\title{
The Effect of Different Brine to Electric Properties of Heavy Oil Emulsion by HLB Scan
}

\author{
Lei Zhang, Zhigang Peng, Shaoxian Wang \\ Shengli Petroleum Engineering Company Limited Drilling Technology Research Institute \& Completion \\ Engineering Key Laboratory, Dongying, China \\ Email: zhanglei16888888@163.com
}

Received 1 April 2014; revised 7 May 2014; accepted 20 May 2014

Copyright (C) 2014 by authors and OALib.

This work is licensed under the Creative Commons Attribution International License (CC BY). http://creativecommons.org/licenses/by/4.0/

c) (i) Open Access

\begin{abstract}
In this study, five kinds of metal salt were selected, and Span 80 and Tween 80 surfactants were selected; they have been used in wide range of hydrophilic-hydrophobic balance (HLB) from 4.3 to 15. The electrical conductivities of heavy oil and different brine emulsion were measured. The objective of this study was to investigate the effect of HLB on the conductivity of heavy oil-brine emulsion. A series of brine-in-heavy oil type (w/o) emulsions with various HLB were prepared. This test used the electric conductivity method to study the physicochemical properties of emulsions. The results indicated that the emulsion conductivity varied widely with the change of HLB value. The conductivity of emulsion containing a sort of metal salt was sequenced as follow: $\mathrm{NiSO}_{4}>$ $\mathrm{Ni}\left(\mathrm{NO}_{3}\right)_{2}>\mathrm{FeSO}_{4}>\mathrm{Co}\left(\mathrm{NO}_{3}\right)_{2}>\left(\mathrm{NH}_{4}\right)_{6} \mathrm{Mo}_{7} \mathrm{O}_{24} \cdot 4 \mathrm{H}_{2} \mathrm{O}$. The conductivity of toluene solution containing Span80 and Tween80 represents exponential relation with HLB. But in the heavy oil-brine emulsion, the relationship between conductivity and HLB was non-linear.
\end{abstract}

\section{Keywords}

Conductivity, Electric Properties, Heavy Oil Emulsion, Hydrophilic-Hydrophobic Balance

\section{Introduction}

The HLB value [1] [2], an empirical parameter representing the content of hydrophilic and hydrophobic groups of a surfactant, has often been employed in the formulation of emulsion products. Emulsifiers for w/o-type emulsions are usually hydrophobic with low HLB values, while emulsifiers with higher HLB number are often used in the preparation of o/w-type emulsions. 
An emulsion is a preparation of one immiscible liquid distributed as small droplets in a second liquid by means of an emulsifying agent. The dispersed liquid is the discontinuous phase and the dispersion medium is the continuous phase. The diameter of the droplets of the dispersed phase lies between 0.1 and $100 \mu \mathrm{m}$. Emulsions are thermodynamically unstable and are subject to change over time. To enhance emulsions' stability and facilitate their formation, emulsifying agents are used. In the simplest Emulsions, the two liquids are water and oil. When oil is the dispersed phase it is known as oil in water $(\mathrm{o} / \mathrm{w})$ emulsion, whereas when water is the dispersed phase it is known as water in oil (w/o) emulsion. Emulsions [3] are also characterized by having a large interfacial area between the liquid phases, allowing a faster exchange process or chemical reaction to take place at the interface. These properties make emulsions useful in foodstuffs, pharmaceuticals, cosmetics, laundry and cleaning agents, and lubricants etc.

The HLB of the surface active agent therefore plays an important role in determining the system properties, and in this work the effect of different brine and surface-active agents with various hydrophobic-lipophilic balance (HLB) to the heavy oil emulsion electric properties was studied. The purpose of this work is to study the change of conductivity about a specific brine/heavy oil emulsion with nonionic surfactant molecules dissolving in the toluene. Using conductivity method to study the structure of w/o microemulsions has many reports [4][7]. Bordi et al. [8] showed that conductivity depends not only on droplet size but also on mean droplet-todroplet distance. Eicke et al. [9] introduced a model to describe the conductivity of w/o microemulsions well below the percolation region. For a particular ion in an electrolyte, $\kappa$ is given by the model of Eicke et al. described briefly below:

$$
\kappa=\frac{z^{2} e v c F}{f}
$$

where $e$ is the elementary charge, $v$ is the number of ions per salt formula, $c$ is the concentration of ions, $F$ is the Faraday constant ( $=\mathrm{N}_{\mathrm{A}} \mathrm{e}$, where $\mathrm{N}_{\mathrm{A}}$ is Avogadro's constant), and $f=6 \pi \eta R_{h}$ is the Stokes frictional factor, where $\eta$ is the solvent viscosity and $R_{h}$ is the hydrodynamic ionic radius. Conductivity measurements have appeared to be a suitable method for study of the interaction of surfactants in such emulsion system as the conductivity of emulsions is sensitive to small changes in the quantity and type of the surfactants. Studies of sedimentation processes, creaming stability, prediction of the inversion points and phase separation utilizing conductivity measurements have been reported [10]. K. I. Al-Malah [11] used the means of conductivity study emulsifying properties of bovine serum albumin in different vegetable oil emulsions. Kobayashi [12] developed the method of conductivity technique to measure emulsifying activity and emulsion stability. However, the relationship between HLB values of the surfactants and the conductivity of emulsion has never been studied. In the present, the emulsifiers used were mixture of Tween80 and Span80 with different ratio to evaluate the effect of HLB value on the electric properties of emulsion.

\section{Materials and Methods}

\subsection{Materials}

Nonionic surfactant polyoxyethylene sorbitan monolaurate (Tween80), Sorbitan monooleate (Span80) were obtained from the Shanghai Chemical Company china, and other reagents were purchased from the Tianjin Chemical reagent Company (China). The heavy oil was used as the continuous phase, the brine was used as the dispersed phase. The properties of Karamay atmospheric heavy oil is shown in Table 1. All emulsions were with distilled water further purified by a Seralpur PRO 90CN deionizing and adsorbing unit.

\subsection{Methods}

\subsubsection{Preparation of Emulsions}

The emulsions were prepared by the apparatus Figure 1. The emulsifiers Span80 and Tween80 were choosed, The required amount of Span80 was dissolved in the oil phase and that of the Tween80 in the aqueou sphase. A series of nine emulsions with HLB values ranging from 4.3 to 15.0 were first prepared by blending together the emulsifiers (Span80 and Tween80) in different ratios, weighing the required amount of each surfactant into clean, stoppered 5-ml volumetric flasks; then the required volumes of were added using an appropriate Hamilton microsyringe. After that, the heavy oil is about $40 \mathrm{~g}$ was added to the beaker, and then the brine of $1.6 \mathrm{ml}$ and 
Table 1. Properties of Karamay atmospheric heavy oil.

\begin{tabular}{cccc}
\hline Items & & Element compose & $\mathrm{m} \%$ \\
\hline Density $\left(20^{\circ} \mathrm{C}\right), \mathrm{g} / \mathrm{m}^{3}$ & 0.9442 & $\mathrm{C}$ & 86.40 \\
Viscosity $\left(100^{\circ} \mathrm{C}\right), \mathrm{mm}^{2} / \mathrm{s}$ & 108.7 & $\mathrm{H}$ & 12.46 \\
Carbon heavy oil, wt\% & 7.0 & $\mathrm{H} /$ Catom ratio & 1.73 \\
Ash content, wt\% & 0.085 & $\mathrm{~S}$ & 0.13 \\
Acid number, mgKOH/g & 5.5 & $\mathrm{~N}$ & 0.41 \\
SARA, m\% & & Metal contend, $\mu \mathrm{g} / \mathrm{g}$ & \\
Saturate, wt\% & 50.4 & $\mathrm{Ni}$ & 11.8 \\
Aromatic, wt\% & 22.2 & $\mathrm{~V}$ & 0.35 \\
Resin, wt\% & 27.2 & $\mathrm{Fe}$ & 10.2 \\
Heptane asphaltene, wt $\%$ & 0.2 & $\mathrm{Ca}$ & 346 \\
\hline
\end{tabular}

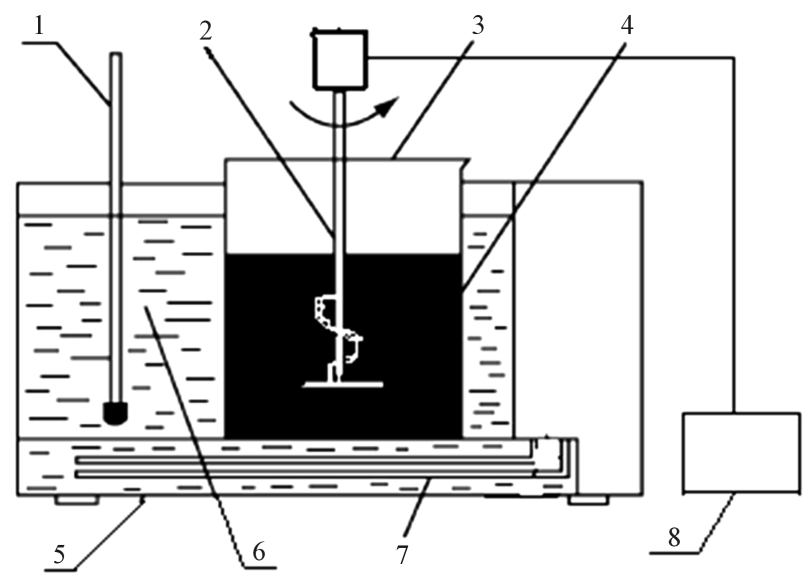

Figure 1. Heavy oil-brine emulsion device. 1 Thermometer; 2 Stirrer; 3 Beaker; 4 Emulsion system; 5 Water bath; 6 Water; 7 Heating tube; 8 Resistance controller.

surfactant was added into the heavy oil using microsyringe. The content of inorganic salt (according to metal account) amounts to $650 \mathrm{ppm}$ of heavy oil. The temperature of reparation of emulsions is $70^{\circ} \mathrm{C}$, the rate of stirring is $800 \mathrm{r} / \mathrm{min}$, and the time is $15 \mathrm{~min}$ to systematically examine the effect of different brine and HLB on the electric properties of emulsion.

\subsubsection{Conductivity Measurements}

The conductivity of emulsions was measured by a Impedance/Gain/Phaseanalyzer conductivity meter (Radio meter shanghai, China). The samples were equilibrated at $30^{\circ} \mathrm{C}$ before measurement. The different emulsion with brine was added into toluene, the emulsion solution of the following concentrations: 3 - 30 g/L, the solution was stirred with magnetic stirrer until a steady conductance value was attained. The purpose of measuring the conductivity of the dispersions was that, since the brine phase present in the Continuous phase of the dispersion system is able to conduct the electric current, the conductivity measurement provides a means of determining the effect brine to the electric properties emulsion.

\section{Results and Discussion}

\subsection{The Influence of HLB Value to Conductivity [6]-[12]}

Span80 and Tween80 which have HLB values from 4.3 to 15 were dissolved in the toluene at the different ratio, and then the conductivities were measured. The effect of HLB on the conductivity was shown in Figure 2. For 


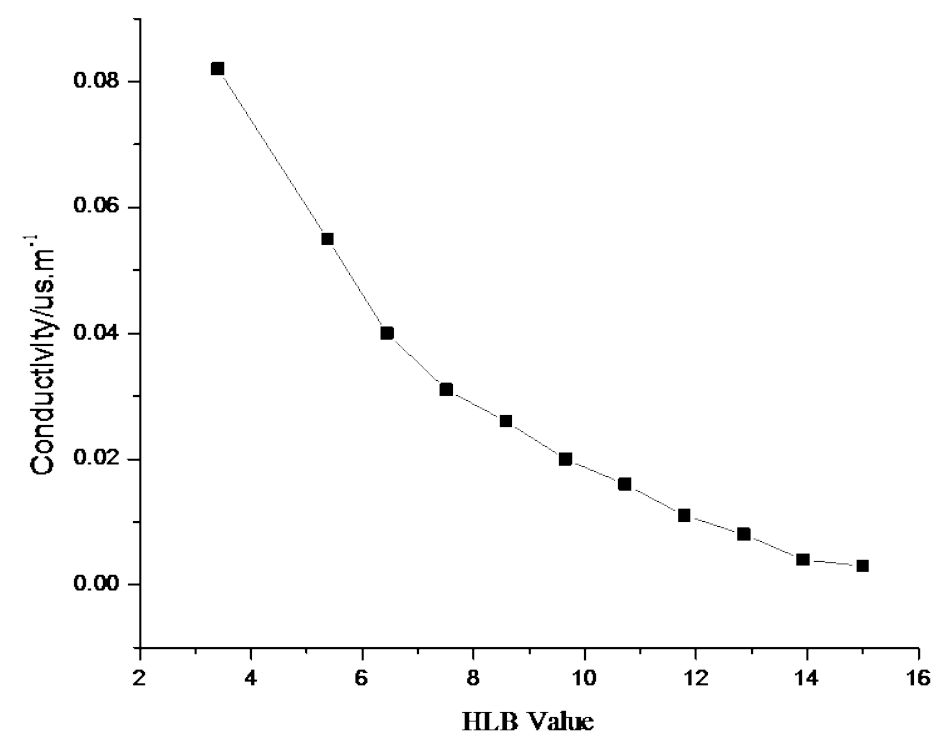

Figure 2. The relation HLB with conductivity in the toluene.

the conductivity of different ratio of Span80 and Tween80 in toluene, the conductivity decreases with increase of HLB, the high HLB has more hydrophilic than low HLB, so The experimental determination results may indicate that the relation HLB with conductivity in the toluene. The experimental data were analysed by the method of exponential regression. The relation conductivity $(k)$ with HLB was calculated as follows: The fact that the experimental HLB can be represented by the conductivity.

$$
k=0.2567 e^{-0.2803 H L B}
$$

\subsection{The Influence of Different Brine to Conductivity of Heavy Oil}

To examine the effect of different brine on the electric properties of emulsion, five sets of emulsions were prepared and characterized. Different concentrations of emulsion in toluene were chosen, covering $2-32 \mathrm{~g} / \mathrm{L}$. the physico-chemical properties of the emulsions will be changed by varying the amounts of added electrolyte, especially the conductivity each group were correlated with the constitutes of brine. A broad variation in specific conductivity of the emulsions was achieved. It is shown in Figure 3.

For each of the sets indicates a non-linear relationship between concentrations and conductivity. The same concentration of quality of Electrolyte has different effect on the conductivity of emulsion. The cation of $\mathrm{NiSO}_{4}$ and $\mathrm{Ni}\left(\mathrm{NO}_{3}\right)_{2}$ is same but the conductivity of emulsion is different. It indicate that anion or cation will affect the conductivity of emulsion, evidently the conductivity of $\mathrm{SO}_{4}^{2-}>\mathrm{NO}_{3}^{-1}$, The conductivities of electrolyte in emulsion were measured, the values of conductivity were sequenced as follow $\mathrm{NiSO}_{4}>\mathrm{Ni}\left(\mathrm{NO}_{3}\right)_{2}>\mathrm{FeSO}_{4}>$ $\mathrm{Co}\left(\mathrm{NO}_{3}\right)_{2}>(\mathrm{NH} 4)_{6} \mathrm{Mo}_{7} \mathrm{O}_{24} \cdot 4 \mathrm{H}_{2} \mathrm{O}$. Figure 3 shows the resulting values for the system during the range of concentration of emulsion. It is seen that brine was added into the heavy oil, and then the conductivity of heavy oil decrease at the quantitative concentration of brine. It is well known that asphaltenes consist of condensed aromatic rings, bound by alkyl chains and containing various functional groups i.e. carboxylic, hydroxyl, ether, ester, aldehyde, ketone, amine and amide. The unit of resin and asphaltene can adsorb electrolyte ions from aqueous solution, and so will be lose a part of polarity and decrease the conductivity of emulsion. This may also explain why effect on the electric properties of emulsion. These results demonstrated clearly that the electrolyte is the main factor affecting the electric properties of emulsion.

\subsubsection{The Effect of $\mathrm{Co}\left(\mathrm{NO}_{3}\right)_{2}$ to the Conductivity of Emulsion with Different HLB}

Cobalt nitrate in the water phase will affect emulsion of physicochemical properties. The results are given in Figure 4, it is known that oil-phase are non-conductive liquids, but resin and asphaltene in the heavy oil are strong polar substance and main electric particles. The change of HLB will affect physicochemical properties of resin, asphaltene and Cobalt nitrate, thereby changing the conductivity of emulsion. Figure 4 shows typical 


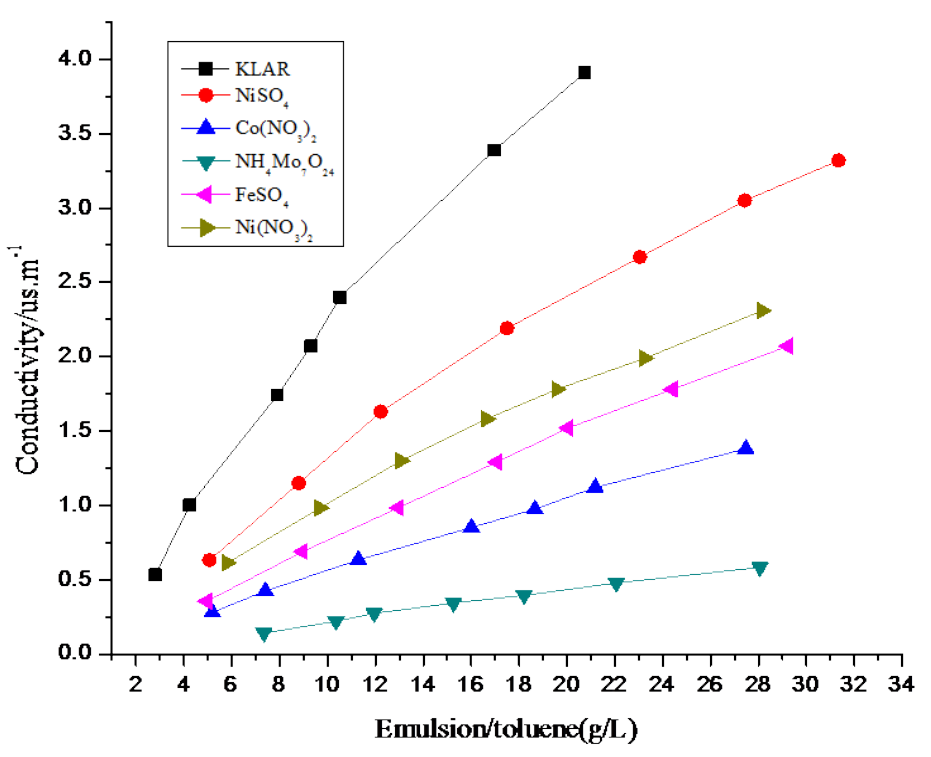

Figure 3. The influence of different brine to conductivity of heavy oil.

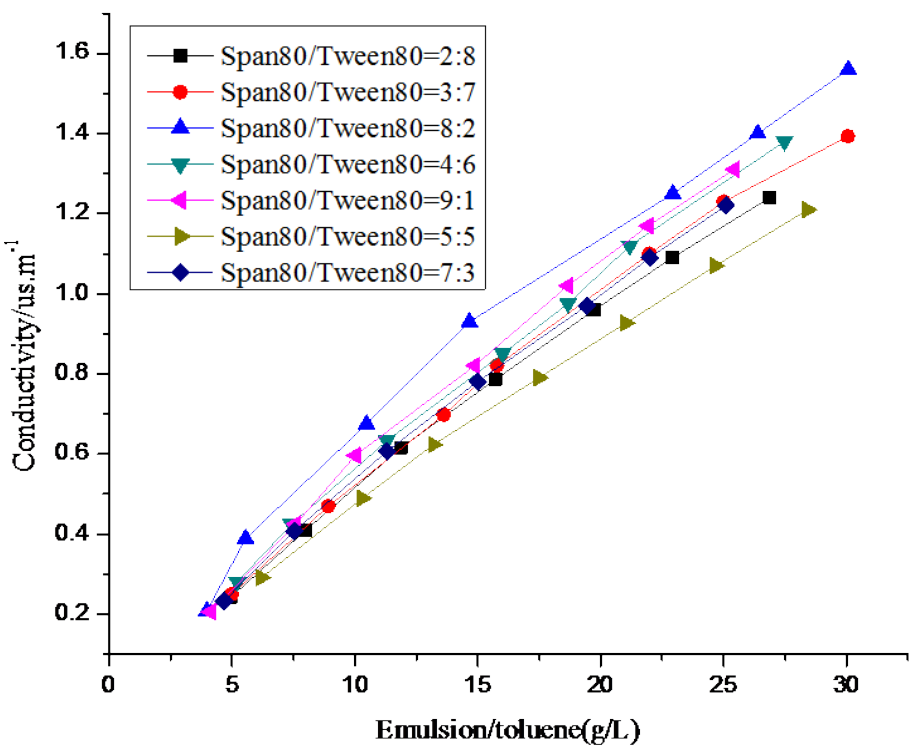

Figure 4. The relation concentration with conductivity at the different ratio Span80:Tween80.

conductivity versus concentration curves. It is observed that a typical conductivity curve shows that the conductivity is the largest at the Span80:Tween80 $=8: 2$ and the least at the Span80:Tween80 $=5: 5$. Moreover at the experimental concentration range, the values of conductivity were sequenced as follow: Span80:Tween80 $=8: 2>$ Span80:Tween80 $=$ 9:1 > Span80:Tween80 $=4: 6>$ Span80:Tween80 $=$ 3:7 > Span80:Tween80 = 7:3 > Span80:Tween80 $=2: 8>$ Span80:Tween80 $=5: 5$. The results show that the HLB value will affect the electric properties of emulsion containing Cobalt nitrate.

\subsubsection{The Effect of (NH4) ${ }_{6} \mathrm{Mo}_{7} \mathrm{O}_{24} \cdot 4 \mathrm{H}_{2} \mathrm{O}$ to the Conductivity of Emulsion with Different HLB}

The conductivity of emulsions is sensitive to small changes in the fraction of the Molybdenum acid ammonium in dispersed phase. The high molecular weight has a higher degree of decreasing the conductivity. Figure 5 shows typical conductivity versus concentration curves. It is observed that a typical conductivity curve shows that the conductivity is the largest at the Span80:Tween $80=4: 6$ and the least at the Span80:Tween80 $=6: 4$, the 


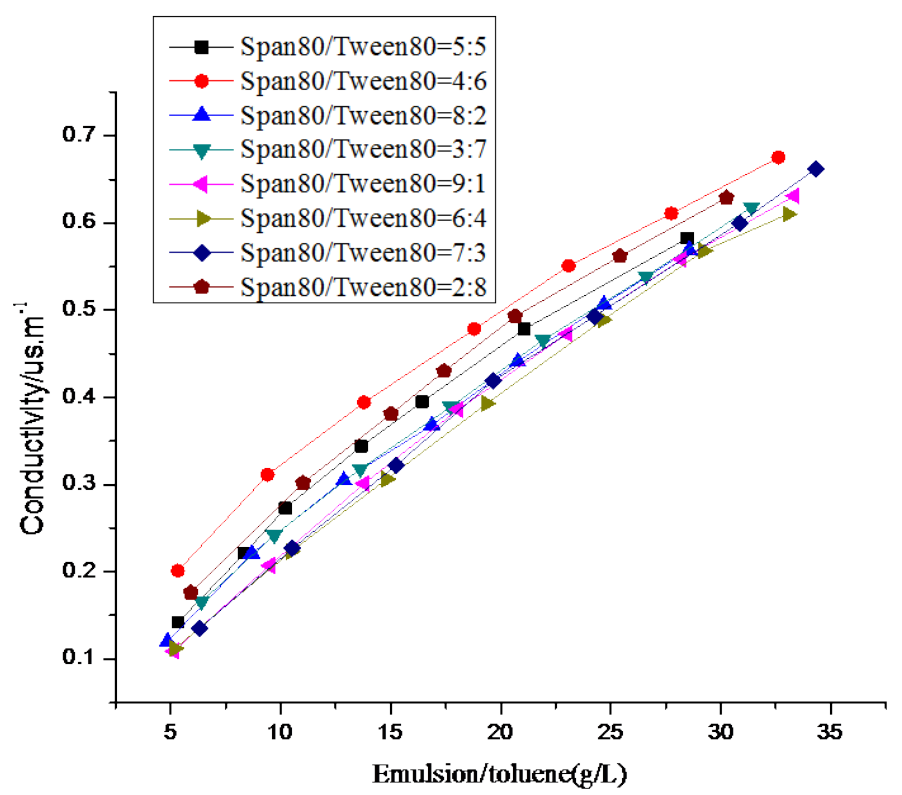

Figure 5. The relation concentration with conductivity at the different ratio Span80:Tween80.

values of conductivity were sequenced as follow: Span80:Tween80 $=4: 6>$ Span80:Tween80 $=2: 8>$ Span80:Tween80 $=5: 5>$ Span80:Tween80 $=3: 7 \approx$ Span80:Tween80 $=$ 8:2 > Span80:Tween80 $=9: 1 \approx$ Span80:Tween80 $=7: 3>$ Span80:Tween80 $=6: 4$. The results of experiment suggested that the conductivities of emulsion have been correlated with their HLB value. Based on classical emulsion science and the HLB concept, most surfactants can be assigned a numerical value representing the hydrophilic-lipophilic characteristics, which determines the type of emulsion. Bancroft's empirical rule states that the phase in which the emulsifier is more soluble is generally the continuous phase. The HLB of the surface-active agent therefore plays an important role in determining the electric properties of emulsion. Such low conductivity is due to the nature of the charge carrier (surfactant stabilized aqueous droplet contenting Molybdenum acid ammonium in a continuous oil phase) and the relatively low ion-exchange rate, owing to the great molecular weight.

\subsubsection{The Effect of $\mathrm{FeSO}_{4}$ to the Conductivity of Emulsion with Different HLB}

The conductivity of emulsion containing ferrous sulphate change with the concentration is different from others. The conductivity of emulsion with different HLB value change is exist difference at the various concentration. The reason of changing may the interaction of ferrous sulphate, surfactant and heavy oil so that deal with the difference. From Figure 6 we can see that When the concentration is less than $15 \mathrm{~g} / \mathrm{L}$, Span80:Tween80 = 8:2 > Span80:Tween $80=5: 5$, When the concentration is more than $15 \mathrm{~g} / \mathrm{L}$, the condition is contrary, at the concentration $20 \mathrm{~g} / \mathrm{L}$, Span80:Tween $80=9: 1$ and Span80:Tween80 $=$ 7:3 have this same phenomenon too. When the concentration is more than $20 \mathrm{~g} / \mathrm{L}$, the conductivity of emulsion in the toluene containing surfactants with various HLB was ranked as: Span80:Tween80 = 5:5 > Span80:Tween80 = 8:2 > Span80:Tween80 = 6:4 > Span80:Tween80 $=2: 8>$ Span80:Tween80 $=7: 3>$ Span80:Tween80 $=9: 1 \approx$ Span80:Tween80 $=3: 7$. Ferrous sulphate was found to have a pronounced different effect on the electric propeties, and illustrates how these ferrous sulphate affect the emulsion in the zone of different concentration, at the present, we have no the suitable reason explain the phenomenon. The mechanism of this process, however, is not quite clear yet. The change of HLB maybe change the ion adsorption state at the film interfaces. Metal salts were added into heavy oil emulsion, the conductivity of emulsion decrease in contrast with the conductivity of heavy oil. This effect indicates possible ion binding by the adsorbed resin, asphaltene or surfactant molecules. Maybe the change of HLB value will affect the ion adsorbent state.

\subsubsection{The Effect of $\mathrm{NiNO}_{3}$ to the Conductivity of Emulsion with Different HLB}

In order to see clearly the distinction of conductivity of different ratio Span80:Tween80, the conductivity of 


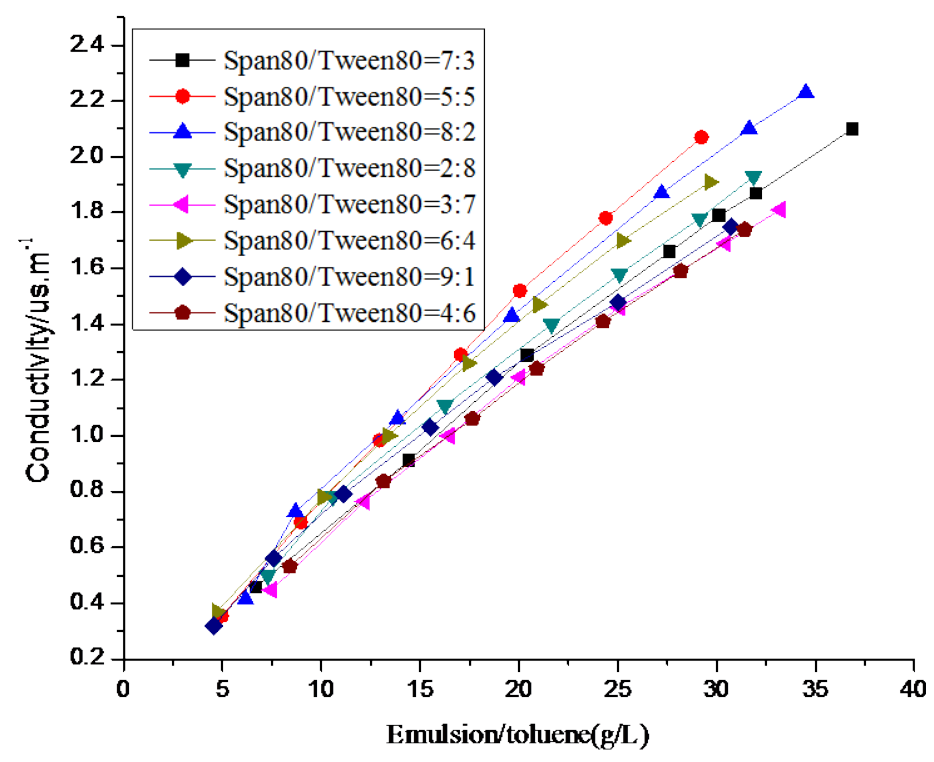

Figure 6. The relation concentration with conductivity at the different ratio Span80:Tween80.

emulsion containing nickel nitrate only line out four sets. Figure 7 shows the conductivity of emulsion with the concentration from 5 to $30 \mathrm{~g} / \mathrm{L}$, the conductivity of emulsion in the toluene containing surfactants with various HLB was ranked as: Span80:Tween80 $=$ 9:1 > Span80:Tween80 = 7:3 > Span80:Tween80 = 5:5 > Span80:Tween80 $=6: 4$. Moreover, the value of conductivity is adjacen comparatively at the concentration is less than $15 \mathrm{~g} / \mathrm{L}$ to the emulsion with the ratio of Span80:Tween80 $=5: 5,6: 4$ and 7:3.

There are surfactant interaction mechanisms of different intensity owing to electrostatic and hydrophobic. Depending on the emulsion system and surfactant concentration, the surfactant may adsorb onto the droplet of brine in the form of individual molecules or small aggregates. The interactions become stronger when the concentration of surfactant increases, and the properties of the system of emulsion change in different ways, depending on the interaction mechanism involved. Because of that the interaction of surfactant-surfactant has to be studied separately for each component on their stability. Therefore the studies of surfactant-surfactant interactions are important from both theoretical and practical point of view. The aim of the present work was to study the surfactant-heavy oil-brine interactions by conductometric. The physicochemical properties of emulsion will change with the change of HLB, especially to the heavy oil-brine emulsion.

\subsection{The Effect of HLB to the Conductivity of Emulsion}

In the present study, the concentration of emulsion is $20 \mathrm{~g} / \mathrm{L}$. We examined the relation HLB and conductivity using emulsions containing surfactants with different HLB values, mainly sorbitan fatty acid esters (Spans) and polyethoxylated sorbitan fatty acid esters (Tweens), in an attempt to evaluate the role of HLB to the conductivity of emulsion In general, the present study indicated that the physicochemical emulsion properties were shown to be significantly influenced by the proportion of emulsion components. Especially to the heavy oil emulsion, brine existing in emulsion affects strongly the electirc properties of emulsion. The results of Figure 8 exhibited that the different brine behaved totally different from one to another. The value and changing trend of conductivity are both different. Increasing the HLB number produces an increase in the relative affinity for the hydrophilic substance, for example in Figure 8 sharp decrease in the conductivity to the nickle nitrate when the HLB is increasing. This decrease is an indication of emulsification. But the conductivity of cobalt nitrate and ferrous sulphate starts to increase gradually and then decreasing with the HLB increasing, and then go on changing with the HLB increasing, from Figure 2 we can see that the relation conductivity with HLB is exponential changing in the toluene. But in the existing of brine and heavy oil the changing trend is very complicated. The emulsion containing nickle nitrate changing trend is different from others, the changing trend of ferrous sulphate, Molybdenum acid ammonium and cobalt nitrate are approximately similar, but the point of extremum is different 


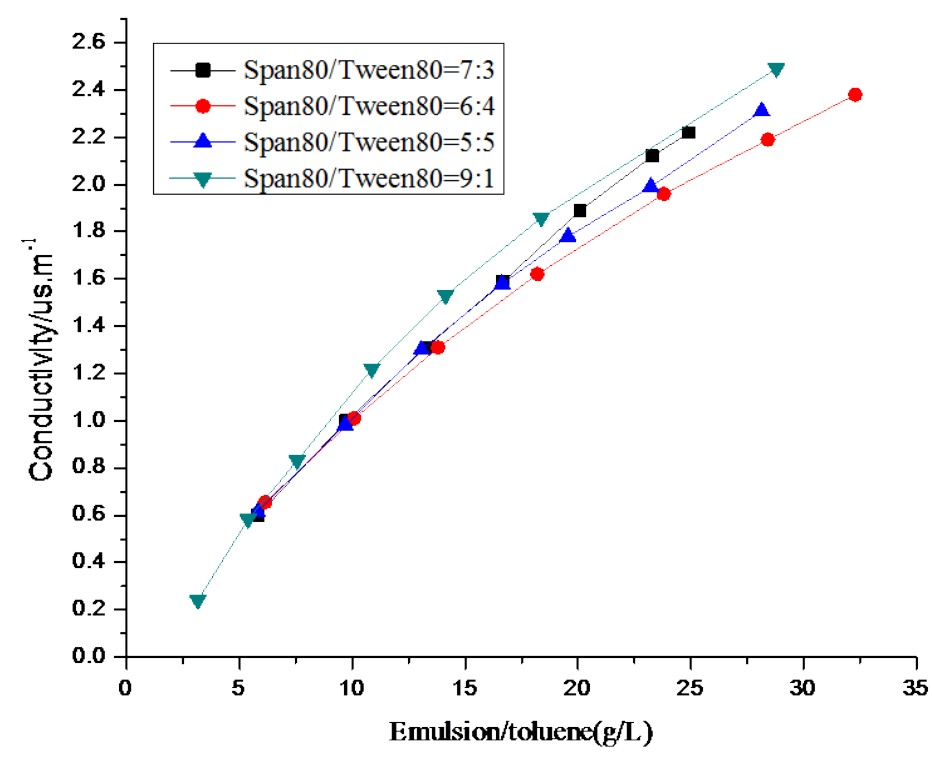

Figure 7. The relation concentration with conductivity at the different ratio Span80:Tween80.

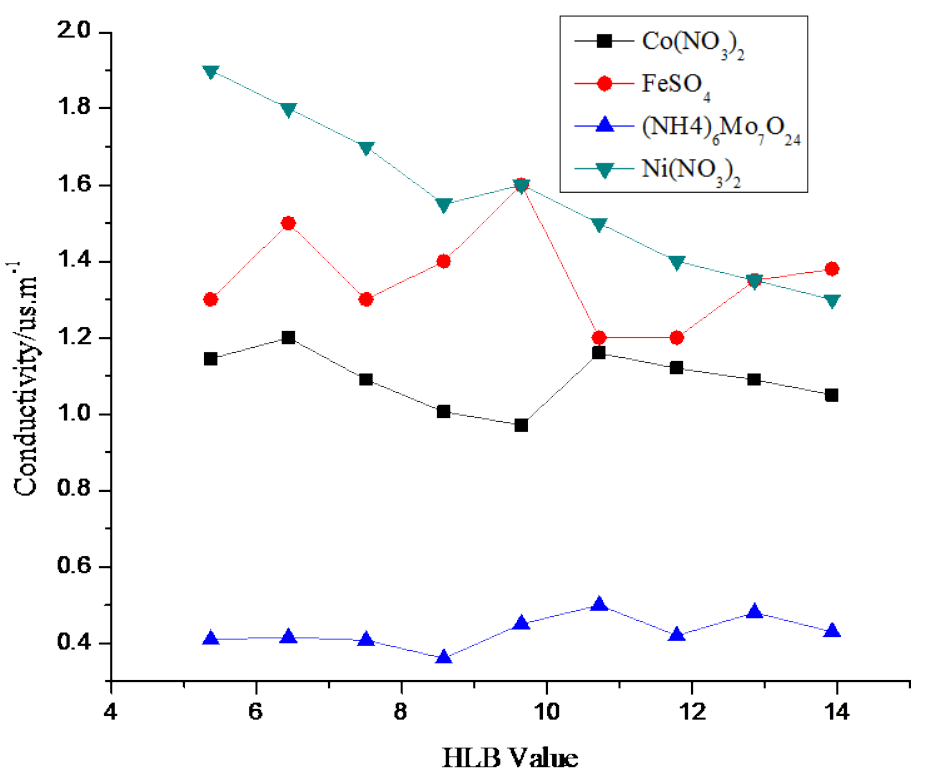

Figure 8. The relation HLB and conductivity at the quantitative concentration of emulsion.

entirety. The change HLB of surfactant to the heavy oil emulsion caused a non-linear change of the conductivity of emulsion. But in the small zone the change of conductivity increased or decreased approximately linearly. It explains that the brine is the main factor affecting the electric properties of emulsion. The brine is happen to interact with the resin, asphaltene in heavy oil and the hydrophilic group of surfactants, and so changing the electric properties of emulsion, nickle, iron, molybdenum, cobalt salts have a great effort in the conductivity of emulsion.

\section{Conclusion}

It has been shown that electrical conductivity measurement is a suitable technique for measurement the change of electirc propeties of heavy oil-brine emulsion. In general, the present study indicated that the physicochemical 
properties of emulsion were shown to be significantly influenced by the emulsion components. Metal salt strongly influenced the electric properties of emulsion. The results exhibited the change of HLB value will affect physicochemical emulsion properties, especially emulsion form with different brine. The results confirmed that the variation of conductivity value as a nonlinear function of HLB value in the heavy oil-brine emulsion. Relationship between HLB and conductivity of emulsion was strongly nonlinear. Metal-salts at lower concentrations had strong effect on the emulsion electirc properties. Conductance is a good parameter to express the ionic strength level. Although clearly the mechanisms involved are complex, there was some correlation between HLB and conductivity. It is obvious that the change of conductivity with the value of HLB presented that interaction among surfactant, metal salts and continuous phase. The condition of interaction shows up through the change of conductivity.

\section{Acknowledgements}

The authors are thankful to Ph.D. Longli Zhang for his invaluable guidance and suggestions.

\section{References}

[1] Hunter, R., Strickland, F. and Kezdy, F. (1981) The Adjuvant Activity of Nonionic Block Polymer Surfactants. Part I. The Role of Hydrophile-Lipophile Balance. The Journal of Immunology, 127, 1244-1250.

[2] Holmberg, K., Jonsson, B., Kronberg, B. and Lindman, B. (2003) Surfactants and Polymers in Aqueous Solution. 2nd Edition, Wiley, New York.

[3] Alava, C. and Saunders, B.R. (2005) Temperature-Responsive Emulsions: The Effect of Added Surfactant. Colloids and Surfaces A: Physicochemical and Engineering Aspects, 270-271, 18-25. http://dx.doi.org/10.1016/j.colsurfa.2005.05.032

[4] Bury, M., Gerhards, J., Emi, W. and Stamm, A. (1995) Application of a New Method Based on Conductivity Measurements to Determine the Creaming Stability of o/w Emulsions. International Journal of Pharmaceutics, $124,183$.

[5] Eicke, H.-F., Borkovec, M. and Das-Gupta, B. (1989) Conductivity of Water-in-Oil Microemulsions: A Quantitative Charge Fluctuation Model. The Journal of Physical Chemistry, 93, 314-317. http://dx.doi.org/10.1021/j100338a062

[6] Lim, K.H., Smith, D.H. and Dispers, J. (1990) Sci. Technol. 11, 529.

[7] Brinks, B.P. and Dong, J. (1998) Emulsions and Equilibrium Phase Behaviour in Silicone Oil + Water + Nonionic Surfactant Mixtures. Colloids and Surfaces A: Physicochemical and Engineering Aspects, 132, 289-301. http://dx.doi.org/10.1016/S0927-7757(97)00183-0

[8] Bury, M., Gerhards, J. and Erni, W. (1991) Monitoring Sedimentation Processes by Conductivity Measurements. International Journal of Pharmaceutics, 76, 207-216. http://dx.doi.org/10.1016/0378-5173(91)90273-Q

[9] Kiekens, F., Vermeire, A., Samyn, N. and Demeester, J. (1997) Optimisation of Electrical Conductance Measurements for the Quantification and Prediction of Phase Separation in o/w-Emulsions, Containing Hydroxypropylmethylcelluloses as emulsifying Agents. International Journal of Pharmaceutics, 146, 239-245. http://dx.doi.org/10.1016/S0378-5173(96)04831-4

[10] Al-Malah, K.I., Azzam, M.O.J. and Omari, R.M. (2000) Emulsifying Properties of BSA in Different Vegetable Oil Emulsions Using Conductivity Technique. Food Hydrocolloids, 14, 485-490.

[11] Kobayashi, K., Kato, A., Fujishige, T. and Matsudomi, N. (1985) Determination of Emulsifying Properties of Some Proteins by Conductivity Measurements. Journal of Food Science, 50, 56-62.

[12] Yang, Y.-W., Wei, A.-C. and Shen, S.-S. (2005) The Immunogenicity-Enhancing Effect of Emulsion Vaccine Adjuvantsis in Dependent of the Dispersion Type and Antigen Release Rate-A Revisit of the Role of the Hydrophile-Lipophile Balance (HLB) Value. Vaccine, 23, 2665-2675. 